\title{
Analysis of SSSS and CCCC Thick Anisotropic Rectangular Plate Using Exact Displacement Function
}

\author{
J. C. Ezeh ${ }^{1}$, O. M. Ibearugbulem ${ }^{2}$, U. C. Anya ${ }^{3}$, H. O. Ozioko ${ }^{4 *}$ \\ ${ }^{1,2,3}$ Department of Civil Engineering Federal University of Technology, Owerri, Imo State, Nigeria \\ ${ }^{4}$ Department of Civil Engineering, Michael Okpara University of Agriculture, Umudike, Abia State, Nigeria
}

DOI: $\underline{10.36348 / \text { sjce.2020.v04i09.003 }}$ | Received: 15.10 .2020 | Accepted: 25.10 .2020 | Published: 05.11 .2020

*Corresponding author: H. O. Ozioko

\section{Abstract}

In this investigation, exact displacement functions were used to analyze thick anisotropic rectangular plates of two boundary conditions; simply supported on all edges (SSSS) and clamped on all edges (CCCC). Third order shear deformation model was employed in the formulation of the total potential energy functional for thick anisotropic rectangular plate. This total potential energy functional was reduced to the governing equation and compatibility equations for thick anisotropic plate. The governing equation and compatibility equations were solved to obtain the general displacement functions. By satisfying the boundary conditions for SSSS and CCCC plates their distinct displacement functions were obtained. These displacement functions were used to obtain the stiffness coefficients $(\mathrm{k}-$ values) for the plates. Minimizing the total potential energy functional with respect to the coefficients of the displacement functions gives the formulas for calculating the values of the coefficients. At this point, the displacements and stresses of the plates were calculated at various angles fiber orientations $\left(0^{\circ}, 15^{\circ}, 30^{\circ}, 45^{\circ}, 60^{\circ}, 75^{\circ}\right.$ and $\left.90^{\circ}\right)$ and various span-tothickness ratios, $\alpha(5,10,20,30,40,50,60,70,80,90$ and 100). The results obtained were close to the results of other scholars.

Keywords: Anisotropic plate; Displacement; governing equation, Compatibility equation; Shear deformation.

Copyright () 2020 The Author(s): This is an open-access article distributed under the terms of the Creative Commons Attribution 4.0 International License (CC BY-NC 4.0) which permits unrestricted use, distribution, and reproduction in any medium for non-commercial use provided the original author and source are credited.

\section{INTRODUCTION}

Anisotropic rectangular plates are one of the most important structural materials used in structural industries and engineering fields such as marine structures, aeronautic, astronautic engineering, etc. This can be attributed to high strength to weight, stiffness to weight, high fatigue strength, excellent corrosion resistance and better tailor ability. Anisotropic materials are much complicated than the isotropic materials due to their in-homogeneity. Hence, it is ideally suited for use in weight sensitive structures and also ideal to understand their static performance in anisotropic plate analysis [1]. The multiple good qualities of anisotropic plates have a great attraction for structural engineers [2].

These literatures adopt refined plate theory just like other thick plate solutions. Thus, non-dimensional thick anisotropic rectangular plate subjected to bending loading and of two boundary conditions; simply supported on all edges (SSSS) and clamped on all edges (CCCC) were analyzed. Iyengar, K.T.S et al., [3] used the method of initial functions to analyze orthotropic rectangular thick plates. Sciuva, M [4] determined the bending, vibration and buckling of simply supported thick multilayered orthotropic plates using a new displacement model. Aydogdu, M [5] derived a new shear deformation theory for laminated composite plates. Noor, A. K et al., [6] assessed the shear deformation theories for multilayered composite plates. Reddy, J et al., [7] derived theories and computational model for composite laminates. Robbins, D.H et al., [8] modeled thick composite using a layer-wise laminate theory. Zhang, Y.X et al., [9] recently developed finite element solution for the analysis of laminated composite plate. Khandan, R et al., [10] developed a laminated composite plate theory. Carrera, E [11] developed theories and finite elements for multilayered anisotropic composite plates and shells. Matsunaga, $\mathrm{H}$ [12] assessed a global higher order deformation theory for laminated composite and sandwich plates. Reddy, J [13] used a simple higher order theory for laminated composite plates. Cho, M [14] used an efficient higher order composite plate theory for general lamination configurations. Poniatovski, V. V [15] worked on the theory of bending of anisotropic plates. Illing, E [16] 
Analyzed the bending of thin anisotropic plates using complex fourth order polynomial differential equation. Vijayakumar, K [17] used Poisson's theory for the analysis of bending of isotropic and anisotropic plates. Vasilenko, A. T [18] determined the bending of an anisotropic elliptic plate on an elastic foundation using the method of successive approximations. Hearmon, R. F. S [19] worked on the bending and twisting of anisotropic plates. Gholami, M [20] worked on the bending analysis of anisotropic functionally graded plates based on three-dimensional elasticity.

In this paper, third order shear deformation theory in Ritz energy method using exact approach was employed to analyze SSSS and CCCC thick anisotropic rectangular plate. With reference to the above discussions on anisotropic and composite plates, it should be noted that the present exact approach method which determined the exact polynomial shear deformations functions of thick anisotropic rectangular plate from the total potential energy functional equation was not employed by any of the work. Hence, it can be stated that the above works are based on the assumed displacement functions and as widely known, assumed displacement functions yields assumed values which may not be wholly relied upon because of possibilities of assuming wrong functions.

\section{THEORETICAL FORMULATION}

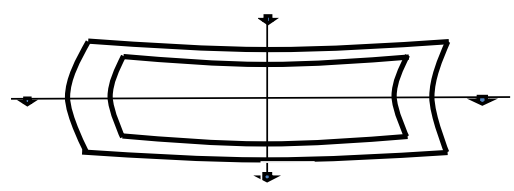

Fig-1a: Deformed rectangular plate showing section A-A and B-B
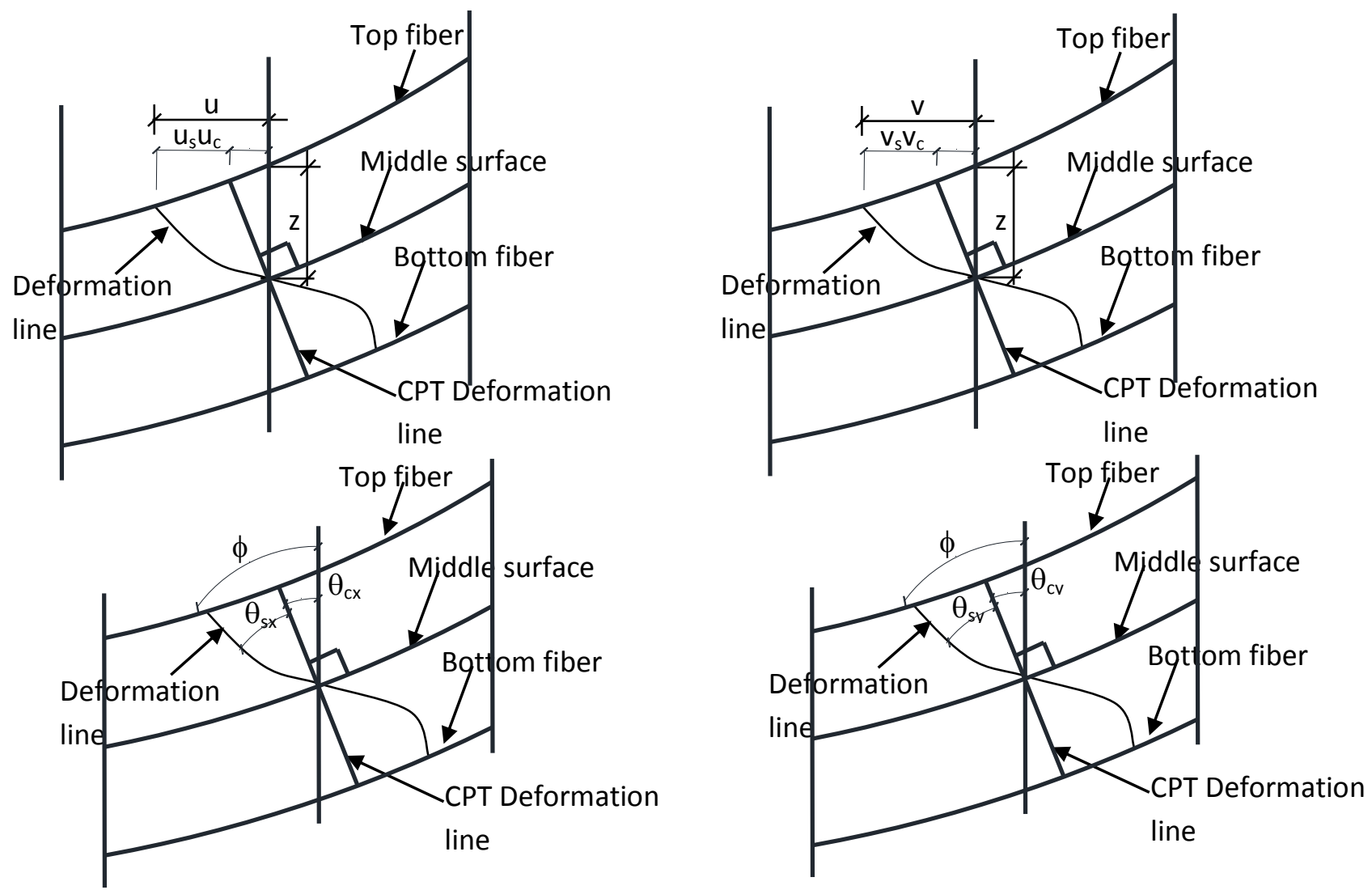

Section A - A

Fig-1b: Deformation of a section of a thick plate

Where: $\mathrm{CPT}=$ Classical Plate Theory, $\phi=$ Total rotation of the middle surface, $\theta_{c x}$ and $\theta_{c y}=$ Classical plate theorem rotation of the middle surface, $\theta_{s x}$ and $\theta_{s y}=$ Angle between the CPT deformation line and the shear deformation line, $\mathrm{u}_{\mathrm{c}}$ and $\mathrm{v}_{\mathrm{c}}=$ In-plane displacement due to classical plate theory and $\mathrm{u}_{\mathrm{s}}$ and $\mathrm{v}_{\mathrm{s}}$ $=$ In-plane displacement due to shear deformation theory. 


\section{Displacement Field}

From Figure-1, the refined plate theory in-plane displacements, $\mathrm{u}$ and $\mathrm{v}$ are defined as presented:

Section B - B

$$
\begin{aligned}
& u=u_{c}+u_{s} \ldots \ldots \ldots \ldots \ldots \ldots \ldots \ldots \ldots 1 \\
& v=v_{c}+v_{s} \ldots \ldots \ldots \ldots \ldots \ldots \ldots \ldots \ldots \ldots .2
\end{aligned}
$$

The non dimensional forms of the orthogonal axes are defined as: $\mathrm{R}=\mathrm{x} / \mathrm{a} ; \mathrm{Q}=\mathrm{y} / \mathrm{b} ; \mathrm{S}=\mathrm{z} / \mathrm{t}$. aspect ratio, denoted as $\beta$ is defined as $\beta=\mathrm{b} / \mathrm{a}$.

The classical part of the in-plane displacements $\mathrm{u}_{\mathrm{c}}$ and $\mathrm{v}_{\mathrm{c}}$ are defined as follows:

$$
u_{c}=-z \theta_{c x}=-z \frac{d w}{d x}=-\frac{S t}{a} \frac{d w}{d R} 3 v_{c}=-z \theta_{c y}=-z \frac{d w}{d y}=-\frac{S t}{b} \frac{d w}{d R}=-\frac{S t}{\beta a} \frac{d w}{d Q} .
$$

Where $\mathrm{w}$, is the out-plane displacement. Transverse displacements $\mathrm{u}_{\mathrm{s}}$ and $\mathrm{v}_{\mathrm{s}}$ are defined as:

$$
\begin{aligned}
& u_{s}=F(z) \theta_{s x} \ldots \ldots \ldots \ldots \ldots \ldots \ldots \ldots \ldots \ldots \\
& v_{s}=F(z) \theta_{s y} \ldots \ldots \ldots \ldots \ldots \ldots \ldots \ldots \ldots \ldots 6
\end{aligned}
$$

Where: $\mathrm{F}(\mathrm{z})$ is the third order shear deformation model defined as:

$$
F(z)=z-\frac{4}{3} \cdot \frac{z^{3}}{t^{2}}=z\left(1-\frac{4}{3}\left[\frac{z}{t}\right]^{2}\right)
$$

The non-dimensional form of the model is:

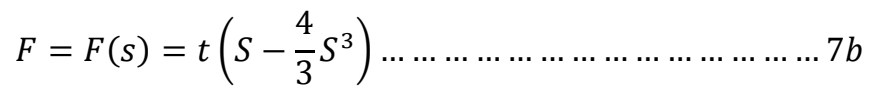

That is:

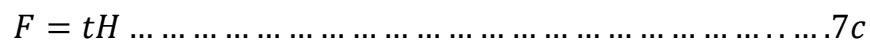

Where:

$$
H=S-\frac{4}{3} S^{3}
$$

Adding Equations 3 and 5 gives:

$$
u=-\frac{S t}{a} \frac{d w}{d R}+F(z) . \emptyset_{x}
$$

Similarly, adding Equations 4 and 6 gives:

$$
v=-\frac{S t}{\beta a} \frac{d w}{d Q}+F(z) \cdot \emptyset_{y}
$$

Substituting Equation 7c into Equations 8a and 8b gives:

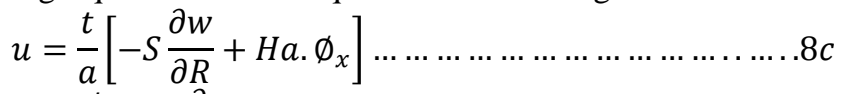

$$
\begin{aligned}
& v=\frac{t}{a \beta}\left[-S \frac{\partial w}{\partial Q}+\beta H a \cdot \emptyset_{y}\right] \text {... }
\end{aligned}
$$

\section{Strain - displacement relations (kinematic relations)}

The strain - displacement relations equations are:

$$
\begin{aligned}
& \varepsilon_{R}=\frac{\partial u}{\partial x}=\frac{\partial u}{a \partial R}=\frac{t}{a^{2}}\left[-S \frac{\partial^{2} w}{\partial R^{2}}+H a \cdot \frac{\partial \phi_{x}}{\partial R}\right] \ldots \ldots \ldots \ldots \ldots \ldots \ldots . . . \cdots \\
& \varepsilon_{Q}=\frac{\partial v}{\partial y}=\frac{\partial v}{a \beta \partial Q}=\frac{t}{\beta^{2} a^{2}}\left[-S \frac{\partial^{2} w}{\partial Q^{2}}+H a \beta \cdot \frac{\partial \phi_{y}}{\partial Q}\right] \ldots \ldots \ldots \ldots \ldots . .10 \\
& \gamma_{R Q}=\varepsilon_{R Q}+\varepsilon_{Q R}=\frac{\partial u}{\partial y}+\frac{\partial v}{\partial x}=\frac{t}{\beta a^{2}}\left[-S \frac{\partial^{2} w}{\partial R \partial Q}+H a \cdot \frac{\partial \phi_{x}}{\partial Q}\right]+\frac{t}{\beta a^{2}}\left[-S \frac{\partial^{2} w}{\partial R \partial Q}+H \beta a \cdot \frac{\partial \phi_{y}}{\partial R}\right] . \text { That is: } \\
& \gamma_{R Q}=\frac{t}{\beta a^{2}}\left[-2 S \frac{\partial^{2} w}{\partial R \partial Q}+H a \cdot\left(\frac{\partial \phi_{x}}{\partial Q}+\beta \cdot \frac{\partial \phi_{y}}{\partial R}\right)\right] \ldots \ldots \ldots \ldots \ldots \ldots 11
\end{aligned}
$$


$\gamma_{R S}=\varepsilon_{R S}+\varepsilon_{S R}=\frac{\partial u}{\partial z}+\frac{\partial w}{\partial x}=\frac{1}{a}\left[-\frac{\partial w}{\partial R}+a \frac{\partial H}{\partial S} \cdot \emptyset_{x}\right]+\frac{1}{a} \frac{\partial w}{\partial R}$. That is:

$$
\gamma_{R S}=\frac{\partial H}{\partial S} \cdot \emptyset_{x}
$$

$\gamma_{Q S}=\frac{\partial H}{\partial S} \cdot \emptyset_{y}=\frac{\partial v}{\partial z}+\frac{\partial w}{\partial y}=\frac{1}{\beta a}\left[-\frac{\partial w}{\partial Q}+\beta a \frac{\partial H}{\partial S} \cdot \emptyset_{y}\right]+\frac{1}{\beta a} \cdot \frac{\partial w}{\partial Q}$. That is:

$$
\gamma_{Q S}=\frac{\partial H}{\partial S} \cdot \emptyset_{y}
$$

\section{Constitutive relations (Stress - Strain Relations)}

$$
\left[\begin{array}{c}
\sigma_{R} \\
\sigma_{Q} \\
\tau_{R Q} \\
\tau_{R S} \\
\tau_{Q S}
\end{array}\right]=\frac{E_{0}}{1-\mu_{12} \mu_{21}}\left[\begin{array}{ccccc}
B_{11} & B_{12} & B_{13} & 0 & 0 \\
B_{21} & B_{22} & B_{23} & 0 & 0 \\
B_{31} & B_{32} & B_{33} & 0 & 0 \\
0 & 0 & 0 & B_{44} & 0 \\
0 & 0 & 0 & 0 & B_{55}
\end{array}\right]\left[\begin{array}{c}
\varepsilon_{R} \\
\varepsilon_{Q} \\
\gamma_{R Q} \\
\gamma_{R S} \\
\gamma_{Q S}
\end{array}\right]
$$

Where:

$\mathrm{E}_{0}$ is the reference Elastic modulus. It can be $\mathrm{E}_{1}$ or $\mathrm{E}_{2} ; m=\operatorname{Cos} \theta ; n=\operatorname{Sin} \theta$

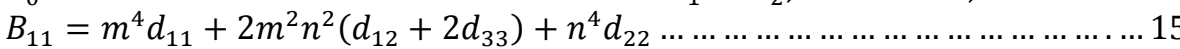

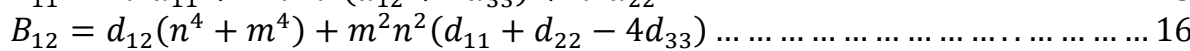

$B_{13}=m^{3} n\left(d_{11}-d_{12}-2 d_{33}\right)+m n^{3}\left(d_{12}-d_{22}+2 d_{33}\right) \ldots \ldots \ldots \ldots \ldots \ldots \ldots \ldots . \ldots \ldots$

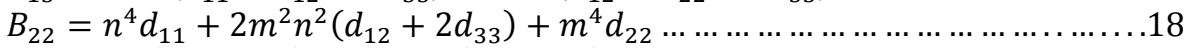

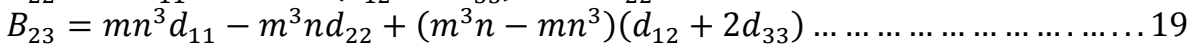

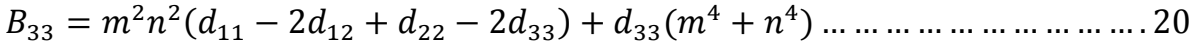

$B_{44}=d_{44} ; B_{55}=d_{55} ; B_{21}=B_{12} ; B_{31}=B_{13} ; B_{32}=B_{23} \ldots \ldots \ldots \ldots \ldots \ldots \ldots \ldots 21$

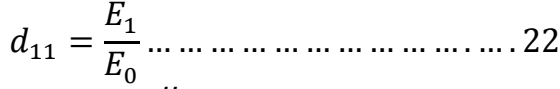

$d_{12}=E_{2} \cdot \frac{\mu_{12}}{E_{0}} \ldots \ldots \ldots \ldots \ldots \ldots \ldots \ldots \ldots \ldots \ldots \ldots \ldots \ldots$

$d_{21}=E_{1} \cdot \frac{\mu_{21}}{E_{0}} \ldots \ldots \ldots \ldots \ldots \ldots \ldots \ldots . \ldots \ldots \ldots$

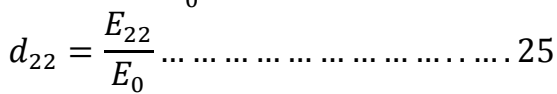

$d_{33}=\frac{G_{12}\left(1-\mu_{12} \mu_{21}\right)}{E_{0}} \ldots \ldots \ldots \ldots . \ldots 26$

$d_{44}=\frac{G_{13}\left(1-\mu_{12} \mu_{21}\right)}{E_{0}} \ldots \ldots \ldots \ldots .27$

$d_{55}=\frac{G_{23}\left(1-\mu_{12} \mu_{21}\right)}{E_{0}} \ldots \ldots \ldots \ldots 28$

Substituting Equations 9 to 13 into Equation 14 gives each stress component as:

$\sigma_{\mathrm{R}}=\frac{\mathrm{E}_{0} \mathrm{t}}{\left[1-\mu_{12} \mu_{21}\right] a^{2}} \cdot\left(\mathrm{B}_{11} \cdot\left[-S \frac{\partial^{2} w}{\partial R^{2}}+H a \cdot \frac{\partial \phi_{x}}{\partial R}\right]+\frac{\mathrm{B}_{12}}{\beta^{2}} \cdot\left[-S \frac{\partial^{2} w}{\partial Q^{2}}+H a \beta \cdot \frac{\partial \phi_{y}}{\partial Q}\right]\right.$ $\left.+\frac{B_{13}}{\beta} \cdot\left[-2 S \frac{\partial^{2} w}{\partial R \partial Q}+H a \cdot\left(\frac{\partial \phi_{x}}{\partial Q}+\beta \cdot \frac{\partial \phi_{y}}{\partial R}\right)\right]\right)$.

$\sigma_{\mathrm{Q}}=\frac{\mathrm{E}_{0} \mathrm{t}}{\left[1-\mu_{12} \mu_{21}\right] a^{2}} \cdot\left(\mathrm{B}_{21} \cdot\left[-S \frac{\partial^{2} w}{\partial R^{2}}+H a \cdot \frac{\partial \phi_{x}}{\partial R}\right]+\frac{\mathrm{B}_{22}}{\beta^{2}} \cdot\left[-S \frac{\partial^{2} w}{\partial Q^{2}}+H a \beta \cdot \frac{\partial \phi_{y}}{\partial Q}\right]\right.$

$$
\left.+\frac{B_{23}}{\beta} \cdot\left[-2 S \frac{\partial^{2} w}{\partial R \partial Q}+H a \cdot\left(\frac{\partial \phi_{x}}{\partial Q}+\beta \cdot \frac{\partial \phi_{y}}{\partial R}\right)\right]\right)
$$

$\tau_{\mathrm{RQ}}=\frac{\mathrm{E}_{0} \mathrm{t}}{\left[1-\mu_{12} \mu_{21}\right] a^{2}} \cdot\left(\mathrm{B}_{31} \cdot\left[-S \frac{\partial^{2} w}{\partial R^{2}}+H a \cdot \frac{\partial \phi_{x}}{\partial R}\right]+\frac{\mathrm{B}_{32}}{\beta^{2}} \cdot\left[-S \frac{\partial^{2} w}{\partial Q^{2}}+H a \beta \cdot \frac{\partial \phi_{y}}{\partial Q}\right]\right.$

$$
\left.+\frac{\mathrm{B}_{33}}{\beta} \cdot\left[-2 S \frac{\partial^{2} w}{\partial R \partial Q}+H a \cdot\left(\frac{\partial \phi_{x}}{\partial Q}+\beta \cdot \frac{\partial \phi_{y}}{\partial R}\right)\right]\right)
$$

$\tau_{\mathrm{RS}}=\frac{\mathrm{E}_{0}}{1-\mu_{12} \mu_{21}} \cdot \mathrm{B}_{44} \cdot\left[\frac{\partial H}{\partial S}\right] \cdot \emptyset_{x}=\frac{\mathrm{E}_{0} \mathrm{t}}{\left[1-\mu_{12} \mu_{21}\right] a^{2}} \cdot \mathrm{B}_{44} \cdot\left[\frac{a^{2}}{\mathrm{t}} \cdot \frac{\partial H}{\partial S}\right] \cdot \emptyset_{x}$ 
$\tau_{\mathrm{QS}}=\frac{\mathrm{E}_{0}}{1-\mu_{12} \mu_{21}} \cdot \mathrm{B}_{55} \cdot\left[\frac{\partial H}{\partial S}\right] \cdot \emptyset_{y}=\frac{\mathrm{E}_{0} \mathrm{t}}{\left[1-\mu_{12} \mu_{21}\right] a^{2}} \cdot \mathrm{B}_{55} \cdot\left[\frac{a^{2}}{\mathrm{t}} \cdot \frac{\partial H}{\partial S}\right] \cdot \emptyset_{y}$

Total potential energy functional

The total potential energy functional is given as:

$$
\begin{gathered}
\Pi=\frac{\mathrm{abt}}{2} \int_{0}^{1} \int_{0}^{1} \int_{-0.5}^{0.5}\left(\sigma_{\mathrm{R}} \varepsilon_{\mathrm{R}}+\sigma_{\mathrm{R}} \varepsilon_{\mathrm{R}}+\tau_{\mathrm{RQ}} \gamma_{\mathrm{RQ}}+\tau_{\mathrm{RS}} \gamma_{\mathrm{RS}}+\tau_{\mathrm{QS}} \gamma_{\mathrm{QS}}\right) \mathrm{dR} \mathrm{dQ} \mathrm{dS} \\
-\mathrm{qab} \int_{0}^{1} \int_{0}^{1} \mathrm{wdRdQ} \ldots \ldots \ldots \ldots \ldots 34
\end{gathered}
$$

Substituting Equations 9 to 13 and Equations 29 to 33 into Equations 34 gives: $\Pi=\frac{\mathrm{abD}_{0}}{2 a^{4}} \cdot \int_{0}^{1} \int_{0}^{1}\left\{\left\{\mathrm{~B}_{11} \cdot\left[\left(\frac{\partial^{2} w}{\partial R^{2}}\right)^{2}-\right.\right.\right.$ $\left.2 g_{2} a \cdot \frac{\partial^{2} w}{\partial R^{2}} \cdot \frac{\partial \phi_{x}}{\partial R}+g_{3} a^{2} \cdot\left(\frac{\partial \phi_{x}}{\partial R}\right)^{2}\right]+\frac{B_{12}}{\beta^{2}} \cdot\left[2\left(\frac{\partial^{2} w}{\partial R \partial Q}\right)^{2}-g_{2} \frac{a}{\beta} \frac{\partial^{2} w}{\partial Q^{2}} \cdot \frac{\partial \phi_{y}}{\partial Q}-g_{2} a \beta^{2} \frac{\partial^{2} w}{\partial R^{2}} \cdot \frac{\partial \phi_{x}}{\partial R}-g_{2} a \cdot \frac{\partial \phi_{x}}{\partial R} \cdot \frac{\partial^{2} w}{\partial Q^{2}}-g_{2} a \beta \cdot \frac{\partial^{2} w}{\partial R^{2}} \cdot \frac{\partial \phi_{y}}{\partial Q}+\right.$ $\left.2 g_{3} a^{2} \beta \cdot \frac{\partial \phi_{x}}{\partial R} \cdot \frac{\partial \phi_{y}}{\partial Q}\right]+\frac{B_{13}}{\beta} \cdot\left[4 \frac{\partial^{2} w}{\partial R \partial Q} \cdot \frac{\partial^{2} w}{\partial R^{2}}-2 g_{2} a \cdot\left(\frac{\partial \phi_{x}}{\partial Q}+\beta \cdot \frac{\partial \phi_{y}}{\partial R}\right) \cdot \frac{\partial^{2} w}{\partial R^{2}}-4 g_{2} a \frac{\partial^{2} w}{\partial R \partial Q} \cdot \frac{\partial \phi_{x}}{\partial R}+2 g_{3} a^{2} \cdot\left(\frac{\partial \phi_{x}}{\partial Q}+\beta \cdot \frac{\partial \phi_{y}}{\partial R}\right) \cdot \frac{\partial \phi_{x}}{\partial R}\right]+$ $\frac{\mathrm{B}_{22}}{\beta^{4}} \cdot\left[\left(\frac{\partial^{2} w}{\partial Q^{2}}\right)^{2}-2 g_{2} a \beta \cdot \frac{\partial^{2} w}{\partial Q^{2}} \cdot \frac{\partial \phi_{y}}{\partial Q}+g_{3} a^{2} \beta^{2} \cdot\left(\frac{\partial \phi_{y}}{\partial Q}\right)^{2}\right]+$

$\frac{B_{23}}{\beta^{3}} \cdot\left[4 \frac{\partial^{2} w}{\partial R \partial Q} \cdot \frac{\partial^{2} w}{\partial Q^{2}}-2 g_{2} a \cdot\left(\frac{\partial \phi_{x}}{\partial Q}+\beta \cdot \frac{\partial \phi_{y}}{\partial R}\right) \cdot \frac{\partial^{2} w}{\partial Q^{2}}-4 g_{2} a \beta \cdot \frac{\partial^{2} w}{\partial R \partial Q} \cdot \frac{\partial \phi_{y}}{\partial Q}+2 g_{3} a^{2} \beta \cdot\left(\frac{\partial \phi_{x}}{\partial Q}+\beta \cdot \frac{\partial \phi_{y}}{\partial R}\right) \cdot \frac{\partial \phi_{y}}{\partial Q}\right]+\frac{B_{33}}{\beta^{2}} \cdot\left[4\left(\frac{\partial^{2} w}{\partial R \partial Q}\right)^{2}-\right.$

$\left.2 g_{2} a \cdot\left(\frac{\partial^{2} w}{\partial R \partial Q} \cdot \frac{\partial \phi_{x}}{\partial Q}+\beta \frac{\partial^{2} w}{\partial R \partial Q} \cdot \frac{\partial \phi_{y}}{\partial R}\right)+g_{3} a^{2} \cdot\left(\left(\frac{\partial \phi_{x}}{\partial Q}\right)^{2}+2 \beta \frac{\partial \phi_{x}}{\partial Q} \cdot \frac{\partial \phi_{y}}{\partial R}+\beta^{2} \cdot\left(\frac{\partial \phi_{y}}{\partial R}\right)^{2}\right)\right]+a^{2} \mathrm{~B}_{44} \cdot\left(\frac{a}{t}\right)^{2} \cdot g_{4} \cdot \emptyset_{x}{ }^{2}+$

$\left.\left.a^{2} \mathrm{~B}_{55} \cdot\left(\frac{a}{t}\right)^{2} \cdot g_{4} \cdot \emptyset_{y}^{2}\right\}-2 \frac{\mathrm{q} a^{4}}{\mathrm{D}_{0}} w\right\} \mathrm{dR} \mathrm{dQ}$. 35

Where:

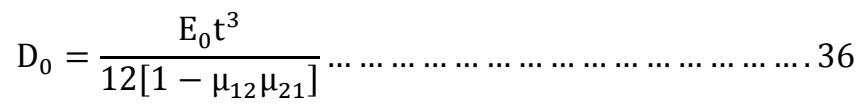

\section{Governing equation and compatibility equations}

Differentiating Equation 35 with respect to $\mathrm{w}, \theta_{\mathrm{x}}$ and $\theta_{\mathrm{y}}$ gives the governing equation and compatibility equations respectively.

$$
\frac{\mathrm{d} \Pi}{\mathrm{dw}}=\frac{\mathrm{d} \Pi}{\mathrm{d} \emptyset_{\mathrm{x}}}=\frac{\mathrm{d} \Pi}{\mathrm{d} \emptyset_{\mathrm{y}}}=037
$$

That is:

$$
\begin{aligned}
& \frac{\mathrm{d} \Pi}{\mathrm{d} w}=\int_{0}^{1} \int_{0}^{1}\left\{\mathrm{~B}_{11} \cdot \frac{\partial^{4} \mathrm{w}}{\partial \mathrm{R}^{4}}+\frac{2}{\beta^{2}} \cdot \mathrm{B}_{\mathrm{xy}} \frac{\partial^{4} \mathrm{w}}{\partial \mathrm{R}^{2} \partial \mathrm{Q}^{2}}+\frac{\mathrm{B}_{22}}{\beta^{4}} \cdot \frac{\partial^{4} \mathrm{w}}{\partial \mathrm{Q}^{4}}+4 \frac{\mathrm{B}_{13}}{\beta} \cdot \frac{\partial^{4} \mathrm{w}}{\partial \mathrm{R}^{3} \partial \mathrm{Q}}+4 \frac{\mathrm{B}_{23}}{\beta^{3}} \cdot \frac{\partial^{4} \mathrm{w}}{\partial \mathrm{R} \partial \mathrm{Q}^{3}}-\frac{\mathrm{g}_{2} \mathrm{a}}{2}\left[2 \mathrm{~B}_{11}+\mathrm{B}_{12}\right] \frac{\partial^{3} \phi_{\mathrm{x}}}{\partial \mathrm{R}^{3}}\right. \\
& -\frac{g_{2} a}{2 \beta^{2}} \cdot B_{x y} \frac{\partial^{3} \phi_{x}}{\partial R \partial Q^{2}}-3 g_{2} a \cdot \frac{B_{13}}{\beta} \frac{\partial^{3} \phi_{x}}{\partial R^{2} \partial Q}-\frac{g_{2} a}{2 \beta^{3}}\left[B_{12}+2 B_{22}\right] \frac{\partial^{3} \phi_{y}}{\partial Q^{3}}-\frac{g_{2} a}{2 \beta} B_{x y} \frac{\partial^{3} \phi_{y}}{\partial R^{2} \partial Q} \\
& \left.-3 g_{2} \text { a. } \frac{B_{23}}{\beta^{2}} \frac{\partial^{3} \phi_{y}}{\partial R \partial Q^{2}}-g_{2} a \cdot B_{13} \cdot \frac{\partial^{3} \phi_{y}}{\partial R^{3}}-\frac{g_{2} a}{\beta^{3}} \cdot B_{23} \cdot \frac{\partial^{3} \phi_{x}}{\partial Q^{3}}-\frac{q^{4}}{D_{0}}\right\} d R d Q=0 \\
& \frac{\mathrm{d} \Pi}{\mathrm{d} \emptyset_{\mathrm{x}}}=\mathrm{B}_{11} \cdot\left[-g_{2} a \cdot \frac{\partial^{3} w}{\partial R^{3}}+g_{3} a^{2} \cdot \frac{\partial^{2} \phi_{x}}{\partial R^{2}}\right]+\frac{\mathrm{B}_{12}}{2 \beta^{2}} \cdot\left[-g_{2} a \beta^{2} \frac{\partial^{3} w}{\partial R^{3}}-g_{2} a \cdot \frac{\partial^{3} w}{\partial R \partial Q^{2}}+2 g_{3} a^{2} \beta \cdot \frac{\partial^{2} \phi_{y}}{\partial R \partial Q}\right] \\
& +\frac{B_{13}}{\beta} \cdot\left[-g_{2} a \cdot \frac{\partial^{3} w}{\partial Q \partial R^{2}}-2 g_{2} a \frac{\partial^{3} w}{\partial Q \partial R^{2}}+2 g_{3} a^{2} \cdot \frac{\partial^{2} \phi_{x}}{\partial R \partial Q}+g_{3} a^{2} \cdot \beta \cdot \frac{\partial^{2} \phi_{y}}{\partial R^{2}}\right] \\
& +\frac{B_{23}}{\beta^{3}} \cdot\left[-g_{2} a \cdot \frac{\partial^{3} w}{\partial Q^{3}}+g_{3} a^{2} \beta \cdot \frac{\partial^{2} \phi_{y}}{\partial Q^{2}}\right]+\frac{B_{33}}{\beta^{2}} \cdot\left[-g_{2} a \cdot \frac{\partial^{3} w}{\partial R \partial Q^{2}}+g_{3} a^{2} \cdot \frac{\partial^{2} \phi_{x}}{\partial Q^{2}}+g_{3} a^{2} \cdot \beta \frac{\partial^{2} \phi_{y}}{\partial R \partial Q}\right] \\
& +a^{2} \mathrm{~B}_{44} \cdot\left(\frac{a}{t}\right)^{2} \cdot g_{4} \cdot \emptyset_{x}=0
\end{aligned}
$$




$$
\begin{aligned}
\frac{\mathrm{d} \Pi}{\mathrm{d} \emptyset_{\mathrm{y}}}=\frac{\mathrm{B}_{12}}{2 \beta^{2}} \cdot\left[-g_{2}\right. & \left.\frac{a}{\beta} \frac{\partial^{3} w}{\partial Q^{3}}-g_{2} a \beta \cdot \frac{\partial^{3} w}{\partial R^{2} \partial Q}+2 g_{3} a^{2} \beta \cdot \frac{\partial^{2} \phi_{x}}{\partial R \partial Q}\right]+\frac{B_{13}}{\beta} \cdot\left[-g_{2} a \cdot \beta \cdot \frac{\partial^{3} w}{\partial R^{3}}+g_{3} a^{2} \cdot \beta \cdot \frac{\partial^{2} \phi_{x}}{\partial R^{2}}\right] \\
& +\frac{\mathrm{B}_{22}}{\beta^{4}} \cdot\left[-g_{2} a \beta \cdot \frac{\partial^{3} w}{\partial Q^{3}}+g_{3} a^{2} \beta^{2} \cdot \frac{\partial^{2} \phi_{y}}{\partial Q^{2}}\right] \\
& +\frac{B_{23}}{\beta^{3}} \cdot\left[-g_{2} a \cdot \beta \cdot \frac{\partial^{3} w}{\partial R \partial Q^{2}}-2 g_{2} a \beta \cdot \frac{\partial^{3} w}{\partial R \partial Q^{2}}+g_{3} a^{2} \beta \cdot \frac{\partial^{2} \phi_{x}}{\partial Q^{2}}+2 g_{3} a^{2} \beta^{2} \cdot \frac{\partial^{2} \phi_{y}}{\partial R \partial Q}\right] \\
& +\frac{\mathrm{B}_{33}}{\beta^{2}} \cdot\left[-g_{2} a \cdot \beta \frac{\partial^{3} w}{\partial R^{2} \partial Q}+g_{3} a^{2} \cdot \beta \frac{\partial^{2} \phi_{x}}{\partial R \partial Q}+g_{3} a^{2} \cdot \beta^{2} \cdot \frac{\partial^{2} \phi_{y}}{\partial R^{2}}\right]+a^{2} \mathrm{~B}_{55} \cdot\left(\frac{a}{t}\right)^{2} \cdot g_{4} \cdot \emptyset_{y} \\
& =0 \ldots \cdots \cdots \cdots \cdots \cdots \cdots \cdots \cdots \cdots \cdots \cdots \cdots \cdots \cdots \cdots \cdots \cdots \cdots \cdots \cdots \cdots
\end{aligned}
$$

Equations 38, 39 and 40 are the governing equation of equilibrium of forces, compatibility equation of displacements in $\mathrm{x}-\mathrm{z}$ plane and compatibility equation of displacements in $\mathrm{y}-\mathrm{z}$ plane respectively.

\section{Solutions of governing equation and compatibility equations}

Solving Equations 38, 39 and 40 gives:

$$
\begin{aligned}
& w=A_{1} h \ldots \ldots \ldots \ldots \ldots . . . . . .41 a \\
& w=\left(\alpha_{0}+\alpha_{1} \mathrm{R}+\alpha_{2} \mathrm{R}^{2}+\alpha_{3} \mathrm{R}^{3}+\alpha_{4} \mathrm{R}^{4}\right)\left(\lambda_{0}+\lambda_{1} \mathrm{Q}+\lambda_{2} \mathrm{Q}^{2}+\lambda_{3} \mathrm{Q}^{3}+\lambda_{4} \mathrm{Q}^{4}\right) . \\
& \phi_{\mathrm{x}}=\frac{\mathrm{A}_{2}}{\mathrm{a}} \cdot \frac{\partial \mathrm{h}}{\partial \mathrm{R}} \ldots \ldots \ldots \ldots \ldots \ldots \ldots+\ldots \ldots+\ldots \ldots \\
& \emptyset_{\mathrm{y}}=\frac{\mathrm{A}_{3}}{\mathrm{a} \beta} \cdot \frac{\partial \mathrm{h}}{\partial \mathrm{Q}}
\end{aligned}
$$

Satisfying the boundary conditions for SSSS and CCCC plates gives their distinct deflection equations respectively as: $w=A_{1} h=\alpha_{3} \alpha_{4}\left(\mathrm{R}-2 \mathrm{R}^{3}+\mathrm{R}^{4}\right)\left(\mathrm{Q}-2 \mathrm{Q}^{3}+\lambda_{4} \mathrm{Q}^{4}\right)\{$ for $S S S S\}$ $41 c$

$w=A_{1} h=\alpha_{3} \alpha_{4}\left(\mathrm{R}^{2}-2 \mathrm{R}^{3}+\mathrm{R}^{4}\right)\left(\mathrm{Q}^{2}-2 \mathrm{Q}^{3}+\lambda_{4} \mathrm{Q}^{4}\right)\{$ for CCCC $\}$ $41 d$

Substituting Equations 41a, 42 and 43 into Equation 35 gives: $\Pi=\frac{a b}{2 a^{4}} \cdot\left\{\left\{\mathrm{B}_{11} \cdot\left[\mathrm{A}_{1}{ }^{2}-2 g_{2} \mathrm{~A}_{1} \mathrm{~A}_{2} \cdot+g_{3} \mathrm{~A}_{2}{ }^{2}\right] k_{1}+\right.\right.$ $\frac{\left(\mathrm{B}_{12}+2 \mathrm{~B}_{33}\right)}{\beta^{2}} \cdot\left[2 \mathrm{~A}_{1}{ }^{2}-g_{2} \mathrm{~A}_{1} \mathrm{~A}_{2}-g_{2} \mathrm{~A}_{1} \mathrm{~A}_{3}\right] \cdot k_{2}+2 \frac{\left[\mathrm{B}_{12}+\mathrm{B}_{33}\right]}{\beta^{2}} g_{3} \mathrm{~A}_{2} \mathrm{~A}_{3} \cdot k_{2}+\frac{\mathrm{B}_{12}}{\beta^{2}} \cdot g_{2}\left[-\frac{\mathrm{A}_{1} \mathrm{~A}_{3}}{\beta^{2}} k_{3}-\mathrm{A}_{1} \mathrm{~A}_{2} \beta^{2} k_{1}\right]+$ $\frac{\mathrm{B}_{33}}{\beta^{2}} \cdot\left[+g_{3} \mathrm{~A}_{2}{ }^{2}+g_{3} \mathrm{~A}_{3}{ }^{2}\right] k_{2}+\frac{B_{13}}{\beta} \cdot\left[4 \mathrm{~A}_{1}{ }^{2}-2 g_{2}\left(\mathrm{~A}_{1} \mathrm{~A}_{2}+\mathrm{A}_{1} \mathrm{~A}_{3}\right)-4 g_{2} \mathrm{~A}_{1} \mathrm{~A}_{2}+2 g_{3}\left(\mathrm{~A}_{2}{ }^{2}+\mathrm{A}_{2} \mathrm{~A}_{3}\right)\right] k_{4}+\frac{\mathrm{B}_{22}}{\beta^{4}} \cdot\left[\mathrm{A}_{1}{ }^{2}-\right.$ $\left.2 g_{2} \mathrm{~A}_{1} \mathrm{~A}_{3}+g_{3} \mathrm{~A}_{3}^{2}\right] k_{3}+\frac{B_{23}}{\beta^{3}} \cdot\left[4 \mathrm{~A}_{1}^{2}-2 g_{2}\left(\mathrm{~A}_{1} \mathrm{~A}_{2}+\mathrm{A}_{1} \mathrm{~A}_{3}\right)-4 g_{2} \mathrm{~A}_{1} \mathrm{~A}_{3}+2 g_{3}\left(\mathrm{~A}_{2} \mathrm{~A}_{3}+\mathrm{A}_{3}{ }^{2}\right)\right] k_{5}+$ $\left.\left.\mathrm{B}_{44} \cdot\left(\frac{a}{t}\right)^{2} \cdot g_{4} \cdot \mathrm{A}_{2}^{2} k_{6}+\frac{\mathrm{B}_{55}}{\beta^{2}} \cdot\left(\frac{a}{t}\right)^{2} \cdot g_{4} \mathrm{~A}_{3}^{2} k_{7}\right\}-2 \mathrm{~A}_{1} \frac{\mathrm{q} a^{4}}{\mathrm{D}_{0}} k_{8}\right\}$

Note:

$\mathrm{k}_{1}=\int_{0}^{1} \int_{0}^{1}\left(\frac{\mathrm{d}^{2} \mathrm{~h}}{\mathrm{dR}^{2}}\right)^{2} \mathrm{dR} \mathrm{dQ} ; \mathrm{k}_{2}=\int_{0}^{1} \int_{0}^{1}\left(\frac{\mathrm{d}^{2} \mathrm{~h}}{\mathrm{dRdQ}}\right)^{2} \mathrm{dR} \mathrm{dQ} ; \mathrm{k}_{3}=\int_{0}^{1} \int_{0}^{1}\left(\frac{\mathrm{d}^{2} \mathrm{~h}}{\mathrm{dQ})^{2}}\right)^{2} \mathrm{dR} \mathrm{dQ}$

$\mathrm{k}_{4}=\int_{0}^{1} \int_{0}^{1}\left(\frac{\mathrm{d}^{2} \mathrm{~h}}{\mathrm{dR}^{2}}\right)\left(\frac{\mathrm{d}^{2} \mathrm{~h}}{\mathrm{dRdQ}}\right) \mathrm{dR} \mathrm{dQ} ; \mathrm{k}_{5}=\int_{0}^{1} \int_{0}^{1}\left(\frac{\mathrm{d}^{2} \mathrm{~h}}{\mathrm{dQ} \mathrm{Q}^{2}}\right)\left(\frac{\mathrm{d}^{2} \mathrm{~h}}{\mathrm{dRdQ}}\right) \mathrm{dR} \mathrm{dQ}$

$\mathrm{k}_{6}=\int_{0}^{1} \int_{0}^{1}\left(\frac{\mathrm{dh}}{\mathrm{dR}}\right)^{2} \mathrm{dR} \mathrm{dQ} ; \mathrm{k}_{7}=\int_{0}^{1} \int_{0}^{1}\left(\frac{\mathrm{dh}}{\mathrm{dQ}}\right)^{2} \mathrm{dR} \mathrm{dQ} ; \mathrm{k}_{8}=\int_{0}^{1} \int_{0}^{1} \mathrm{hdR} \mathrm{dQ}$

To obtain the quasi equations of equilibrium of forces and quasi compatibility equations, Equation 44 must be differentiated with respect to $\mathrm{A} 1, \mathrm{~A} 2$ and $\mathrm{A} 3$. That is:

$\frac{\mathrm{d} \Pi}{\mathrm{dA}_{1}}=\frac{\mathrm{d} \Pi}{\mathrm{dA}_{2}}=\frac{\mathrm{d} \Pi}{\mathrm{dA}_{3}}=0$

$\frac{\mathrm{d} \Pi}{\mathrm{dA} \mathrm{A}_{1}}=\mathrm{L}_{11} \mathrm{~A}_{1}-\mathrm{L}_{12} \mathrm{~A}_{2}-\mathrm{L}_{13} \mathrm{~A}_{3}-\frac{\mathrm{q} a^{4}}{\mathrm{D}_{0}} \mathrm{k}_{8}=0$

$\frac{\mathrm{d} \Pi}{\mathrm{dA}_{2}}=\mathrm{L}_{12} \mathrm{~A}_{1}-\mathrm{L}_{22} \mathrm{~A}_{2}-\mathrm{L}_{23} \mathrm{~A}_{3}=0$

$\frac{\mathrm{d} \Pi}{\mathrm{dA}}=\mathrm{L}_{13} \mathrm{~A}_{1}-\mathrm{L}_{23} \mathrm{~A}_{2}-\mathrm{L}_{33} \mathrm{~A}_{3}=0$ 
Where:

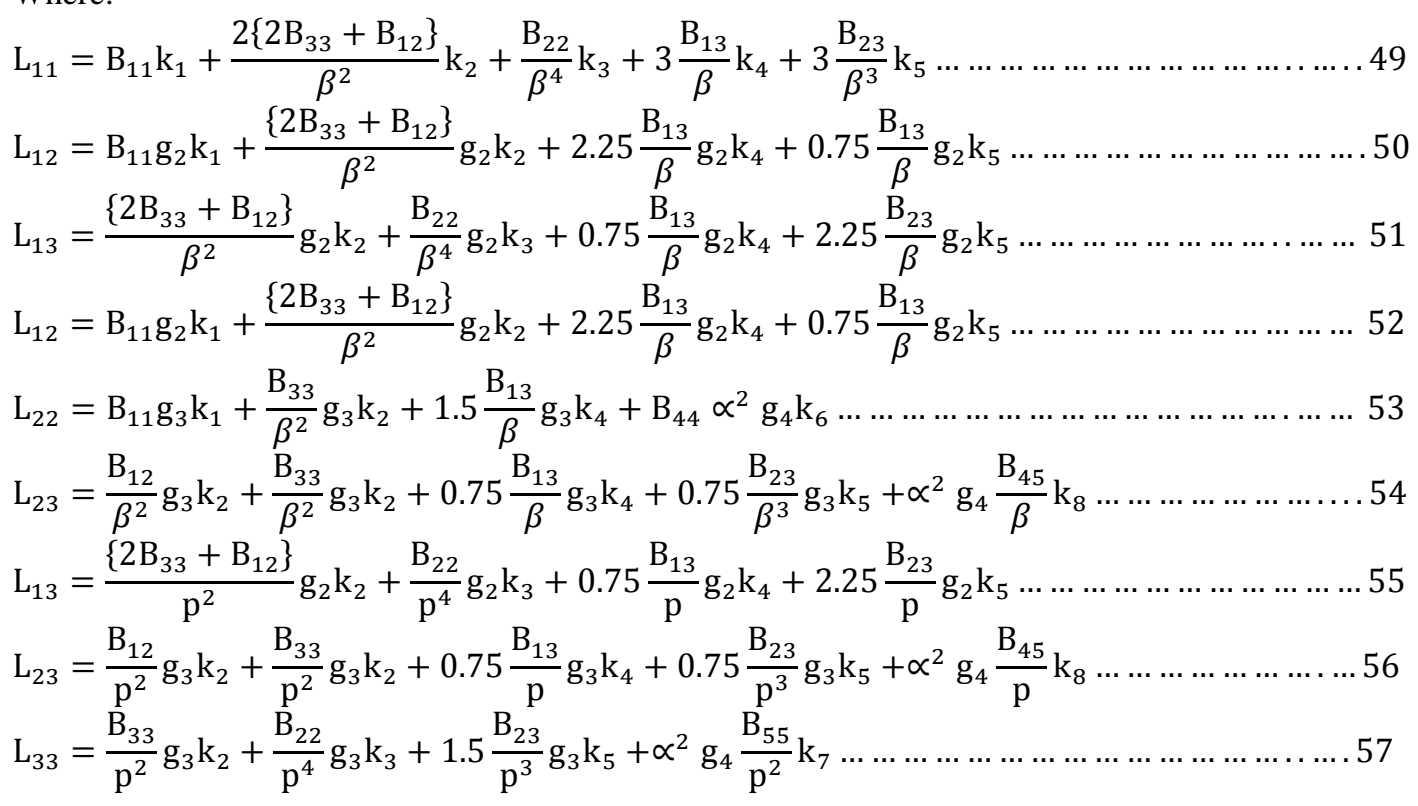

Solving Equations 47 and 48 simultaneously gives:

$$
\begin{aligned}
& A_{2}=\left(\frac{L_{12} L_{33}-L_{13} L_{23}}{L_{22} L_{33}-L_{23} L_{23}}\right) A_{1}=P_{2} A_{1} \\
& A_{3}=\left(\frac{L_{13} L_{22}-L_{12} L_{23}}{L_{22} L_{33}-L_{23} L_{23}}\right) A_{1}=P_{3} A_{1}
\end{aligned}
$$

Substituting Equations 57 and 58 into Equation 46 gives:

$$
\mathrm{A}_{1}=\frac{\mathrm{q} a^{4}}{\mathrm{D}_{0}} \cdot \frac{\mathrm{k}_{8}}{\left(\mathrm{~L}_{11}-\mathrm{L}_{12} P_{2}-\mathrm{L}_{13} P_{3}\right)}=\frac{\mathrm{q} a^{4}}{\mathrm{D}_{0}} \cdot \mathrm{k}_{9}
$$

\section{Formulas for analysis}

Substituting Equation 60 into Equation 41a and substituting Equation 36 into the resulting equation and simplifying gives:

$$
w \frac{\mathrm{E}_{0} \mathrm{t}^{3}}{\mathrm{q} a^{4}}=12\left[1-\mu_{12} \mu_{21}\right] . \mathrm{k}_{9} h .
$$

Substituting Equations 41a, 42 and 43 into Equations 8c, 8d, 29, 30, 31, 32 and 33, where appropriate and simplifying gives:

$$
\begin{aligned}
& u \frac{\mathrm{E}_{0}}{\mathrm{q} a}\left(\frac{\mathrm{t}}{\mathrm{a}}\right)^{2}=12\left[1-\mu_{12} \mu_{21}\right] \cdot \mathrm{k}_{9} \cdot\left[-S+H P_{2}\right] \cdot \frac{\partial \mathrm{h}}{\partial \mathrm{R}} . \\
& v \frac{\mathrm{E}_{0}}{q a}\left(\frac{\mathrm{t}}{\mathrm{a}}\right)^{2}=12\left[1-\mu_{12} \mu_{21}\right] \cdot \frac{\left[-S+H \cdot P_{3}\right]}{\beta} \cdot \frac{\partial h}{\partial Q} \cdot \mathrm{k}_{9} \\
& \frac{\sigma_{\mathrm{R}}}{\mathrm{q}}\left(\frac{t}{a}\right)^{2}=12 . \mathrm{k}_{9}\left(\mathrm{~B}_{11}\left[H P_{2}-S\right] \frac{\partial^{2} h}{\partial R^{2}}+\frac{\mathrm{B}_{12}}{\beta^{2}}\left[H P_{3}-S\right] \frac{\partial^{2} h}{\partial Q^{2}}+\frac{B_{13}}{\beta} H\left(P_{2}+P_{3}-2 S\right) \frac{\partial^{2} h}{\partial R \partial Q}\right) \ldots \\
& \frac{\sigma_{\mathrm{Q}}}{\mathrm{q}}\left(\frac{t}{a}\right)^{2}=12 . \mathrm{k}_{9} \cdot\left(\mathrm{B}_{21}\left[H P_{2}-S\right] \frac{\partial^{2} h}{\partial R^{2}}+\frac{\mathrm{B}_{22}}{\beta^{2}}\left[H P_{3}-S\right] \frac{\partial^{2} h}{\partial Q^{2}}+\frac{B_{23}}{\beta} H\left(P_{2}+P_{3}\right)-2 S \frac{\partial^{2} h}{\partial R \partial Q}\right) \ldots \\
& \frac{\tau_{\mathrm{RQ}}}{\mathrm{q}}\left(\frac{t}{a}\right)^{2}=12 \mathrm{k}_{9} \cdot\left(\mathrm{B}_{31} \cdot\left[H P_{2}-S\right] \frac{\partial^{2} h}{\partial R^{2}}+\frac{\mathrm{B}_{32}}{\beta^{2}} \cdot\left[H P_{3}-S\right] \frac{\partial^{2} h}{\partial Q^{2}}+\frac{\mathrm{B}_{33}}{\beta} \cdot H\left(P_{2}+P_{3}-2 S\right) \frac{\partial^{2} h}{\partial R \partial Q}\right) \\
& \overline{\tau_{\mathrm{RS}}}=\frac{\tau_{\mathrm{RS}}}{\mathrm{q}}\left(\frac{t}{a}\right)^{3}=12 \mathrm{k}_{9}\left(\mathrm{~B}_{44} \cdot P_{2} \cdot \frac{\partial H}{\partial S}\right) \cdot \frac{\partial \mathrm{h}}{\partial \mathrm{R}} \text {. } \\
& \overline{\tau_{\mathrm{QS}}}=\frac{\tau_{\mathrm{QS}}}{\mathrm{q}}\left(\frac{t}{a}\right)^{3}=12 . \mathrm{k}_{9} \cdot\left(\mathrm{B}_{55} \cdot \frac{P_{3}}{\beta} \cdot \frac{\partial H}{\partial S}\right) \cdot \frac{\partial \mathrm{h}}{\partial \mathrm{Q}} .
\end{aligned}
$$




\section{Example Problems}

The numerical values for typical anisotropic rectangular thick plate In-plane displacements, $\mathrm{u}$ and $\mathrm{v}$, out-plane displacement (central deflection), w, in-plane stresses, $\sigma_{\mathrm{x}}, \sigma_{\mathrm{y}}$ and $\tau_{\mathrm{xy}}$, and out-plane stresses, $\tau_{\mathrm{xz}}$ and $\tau_{\mathrm{yz}}$, were determined for angles fiber orientations of $0^{0}$, $15^{\circ}, 30^{\circ}, 45^{\circ}, 60^{\circ}, 75^{\circ}$ and $90^{\circ}$ at span to thickness ration $(\alpha), 5,10,20,30,40,50,60,70,80,90$ and 100 for plate of two boundary conditions; SSSS and CCCC that are subjected to bending loading. The plate was analyzed at various meaningful points along the length, width and depth axis. For SSSS plate: in-plane displacements, $\mathrm{u}$ and $\mathrm{v}$, were analyzed at coordinates $(\mathrm{x}=0.5, \mathrm{y}=0.5, \mathrm{z}=0.5) \quad ; \quad$ transverse displacement, $\mathrm{w}$, was analyzed at coordinate $(\mathrm{x}=$ $0.5, \mathrm{y}=0.5, \mathrm{z}=0.5)$, In-plane normal stresses, $\sigma_{\mathrm{x}}$ and $\sigma_{\mathrm{y}}$, were analyzed at coordinates $(\mathrm{x}=0.5, \mathrm{y}=$ $0.5, \mathrm{z}=0.5)$, in-plane shear stress, $\tau_{\mathrm{xy}}$, were analyzed at coordinates $(\mathrm{x}=0, \mathrm{y}=0, \mathrm{z}=0.5)$, out-plane shear stresses, $\tau_{\mathrm{xz}}$ and $\tau_{\mathrm{yz}}$, were analyzed at coordinates $(\mathrm{x}=0, \mathrm{y}=0.5, \mathrm{z}=0.5) \quad$ and $\quad(\mathrm{x}=0.5, \mathrm{y}=$ $0, \mathrm{z}=0.5)$ respectively. For CCCC plate: in-plane displacements, $\mathrm{u}$, was analyzed at coordinate $(\mathrm{x}=$ $0.2, \mathrm{y}=0.5, \mathrm{z}=0.5)$; in-plane displacement, $\mathrm{v}$, was analyzed at coordinate $(\mathrm{x}=0.5, \mathrm{y}=0.2, \mathrm{z}=0.5)$; transverse displacement, $w$, was analyzed at coordinate $(\mathrm{x}=0.5, \mathrm{y}=0.5, \mathrm{z}=0.5) \quad ; \quad$ In-plane normal stresses, $\sigma_{\mathrm{xx}}, \sigma_{\mathrm{yy}}$ and in-plane shear stress, $\tau_{\mathrm{xy}}$, were analyzed at coordinates $(\mathrm{x}=0.2, \mathrm{y}=0.2, \mathrm{z}=0.5)$; while out-plane shear stresses, $\tau_{\mathrm{xz}}$ and $\tau_{\mathrm{yz}}$, were analyzed at coordinates $(\mathrm{x}=0.2, \mathrm{y}=0.5, \mathrm{z}=0.5)$ and $(\mathrm{x}=0.5, \mathrm{y}=0.2, \mathrm{z}=0.5)$ respectively. The plate is subjected to uniformly distributed load. The following non dimensional forms applied by [21, 22, $23,24,25]$ were used to present the results: $[\overline{\mathrm{w}}=$ $\mathrm{w} \frac{\mathrm{E}_{0} \mathrm{t}^{3}}{\mathrm{q} a^{4}} \mathrm{x} 100 ; \overline{\mathrm{u}}, \overline{\mathrm{v}}=\mathrm{u}, \mathrm{v} \frac{\mathrm{E}_{0} \mathrm{t}^{2}}{\mathrm{q} a^{3}} ; \overline{\sigma_{\mathrm{xx}}}, \overline{\sigma_{\mathrm{yy}}}, \overline{\tau_{\mathrm{xy}}}=$ $\left.\frac{\sigma_{\mathrm{x}}, \sigma_{\mathrm{y}}, \tau_{\mathrm{xy}}}{\mathrm{q}} \cdot\left(\frac{t}{a}\right)^{2} ; \overline{\tau_{\mathrm{xz}}}, \overline{\tau_{\mathrm{yz}}}=\frac{\tau_{\mathrm{xz}}, \tau_{\mathrm{yz}}}{\mathrm{q}} \cdot\left(\frac{t}{a}\right).\right]$ The material properties used for Tables 1, 2 and 3 have the following given parameters: $\left(\mathrm{E}_{1} / \mathrm{E}_{2}=25, \mathrm{G}_{12} / \mathrm{E}_{2}=\right.$ $\left.0.5, \mathrm{G}_{13} / \mathrm{E}_{2}=0.5, G_{23} / \mathrm{E}_{2}=0.2 ; \mathrm{v}_{12}=0.25\right)$

while Table 4 used these parameters: $\left(E_{1}=E_{2}=\right.$ 210GPa; $\mathrm{v}_{12}=0.3 ; \mathrm{G}=\frac{E}{2(1+\mu)}$.)

i. Analyze an orthotropic thick square SSSS plate with the following information: $\left(\mathrm{E}_{1}=\right.$ $\left.\mathrm{E}_{2}=210 \mathrm{GPa}, \mathrm{v}_{12}=0.3, \mathrm{G}=\frac{E}{2(1+\mu)} \cdot\right)$

ii. Analyze an orthotropic thick square CCCC plate with the following information: $(\mathrm{E} 1=$ 25; $\mathrm{E} 2=1 ; \mathrm{G} 12=0.5 ; \mathrm{G} 13=$ $0.5 ; \mathrm{G} 23=0.2, \mu 12=0.25$ )

\section{RESULTS AND DISCUSSIONS Presentation of Results}

This study investigated SSSS and CCCC thick anisotropic rectangular plates and the following results were determined.

\section{Results of Numerical Problems}

The numerical values for typical anisotropic rectangular thick plate In-plane displacements, $u$ and $v$, out-plane displacement (central deflection), w, in-plane stresses, $\sigma_{\mathrm{x}}, \sigma_{\mathrm{y}}$ and $\tau_{\mathrm{xy}}$, and out-plane stresses, $\tau_{\mathrm{xz}}$ and $\tau_{\mathrm{yz}}$, as determined are presented on Tables (1) to (3).

\section{Example problem of typical anisotropic rectangular thick plate with different boundary conditions}

The method developed was deployed to solve typical anisotropic rectangular thick plate problems. The problems chosen have also been solved by other authors for SSSS rectangular plate at zero degrees angle fiber orientation. The values gotten were compared with those from other authors as shown on Table-4. Below are discussions made based on the results.

\section{Numerical values of SSSS plate at angle fiber orientation of $0^{0}$}

From Table-1, it is observed that out-plane displacement $(\mathrm{w})$ decreases in values as the thickness of the plate decreases. This decrease is very visible at thick plate zone ( $\alpha=5$ t0 10) but gradually diminishes as thickness of the plate decreases. The in-plane displacements (u \&v) yielded negative values which decrease as the thickness of the plate decreases. The decrease was more at the thick plate zone but gradually reduces at the thin plate zone. This shows that the outplane displacement are more effective on thick plate than thin plate. The in-plane stresses, $\overline{\sigma_{x x}}$ and $\tau_{x y}$, decrease in values as the plate decreases in thickness. A closer look will show a sharp decrease at thick plate section and a slight decrease at thin plate section. The in-plane stress $\overline{\sigma_{y y}}$, out-plane stresses $\tau_{x z}$ and $\tau_{y z}$, increase in values as the plate thickness decreases. This increase and decrease of the stresses and displacements are due to the anisotropic nature of the plate.

\section{Numerical values of $\mathrm{CCCC}$ plate at angle fiber orientation of $0^{0}$}

From Table-2, it is observed that out-plane displacement values, $\bar{w}$, decreases as thickness of the plate decreases. The values given by the in-plane displacements $\mathrm{u}$, and $\mathrm{v}$, are in the negative coordinates and they decrease as the thickness of the plate decreases. In-plane stresses, $\overline{\sigma_{\mathrm{xx}}}$ and $\overline{\sigma_{\mathrm{yy}}}$ values are also in the negative coordinates and they increase as the thickness of the plate increases. In-plane stress, $\overline{\tau_{x y}}$ and out-plane stress, $\overline{\tau_{\mathrm{yz}}}$ decrease in values as the thickness of the plate decreases while the out-plane stress, $\overline{\tau_{x z}}$, 
increases in values as the thickness of the plate decreases.

Hence, it can be stated that, for CCCC plate at angle fiber orientation of $0^{\circ}$, displacements and stresses are either increasing or decreasing in values as the plate thickness decreases. This shows how anisotropic the plate is in nature.

\section{Numerical values of $\mathrm{CCCC}$ plate at angle fiber orientation of $15^{0}$}

Table-2 shows that out-plane displacement values, $\bar{w}$, decreases in values as the thickness of the plate decreases. The in-plane displacements $\mathrm{u}$, and $\mathrm{v}$, gave values at the negative coordinates, these values decease as the thickness of the plate decreases. The Inplane stresses, $\overline{\sigma_{x x}}$ and $\overline{\sigma_{y y}}$ values are also in the negative coordinates and they increase as the thickness of the plate decreases. In-plane stress, $\overline{\tau_{x y}}$ and out-plane stresses, $\overline{\tau_{\mathrm{xz}}}$ and $\overline{\tau_{\mathrm{yz}}}$, decrease in values as the thickness of the plate decreases,

Also, for CCCC plate at angle fiber orientation of $15^{\circ}$, displacements and stresses are either increasing or decreasing in values as the plate thickness decreases. This explains the anisotropic nature of the plate.

\section{Comparison of present study results with those from various authors.}

The aspect ratio was inverted to correspond with the one used by other authors $(1 / \beta=\mathrm{a} / \mathrm{b}=$ 0.5 , i. e. $\beta=b / a=2)$. This aspect ratio, $(\beta=2)$ was employed to determine the values of displacements and stresses as shown on Table-4.

The percentage differences between present study and Ghugal and Sayyad (2010) for displacement $(\overline{\mathrm{w}})$ and in-plane stress $\left(\overline{\sigma_{\mathrm{xx}}}\right)$ are $4.97 \%$ and $2.98 \%$. Also, the percentage differences between present study and Ghugal and Sayyad (2010) for stresses $\left(\overline{\tau_{x z}}, \overline{\sigma_{y y}}\right.$ and $\left.\overline{\tau_{x y}}\right)$ are $-9.87 \%, 27.49 \%$ and $-20.7 \%$ respectively. This shows that, present study displacement $(\overline{\mathrm{W}})$ and in-plane stress $\left(\overline{\sigma_{\mathrm{xx}}}\right)$ are closely related to those obtained by Ghugal and Sayyad (2010) but mildly over-estimated the in-plane stresses $\left(\overline{\sigma_{\mathrm{yy}}}\right)$ and $\left(\overline{\tau_{\mathrm{xy}}}\right)$. The percentage differences between the present study and Murthy (1981) results for displacement $(\overline{\mathrm{W}})$ and stresses $\left(\overline{\sigma_{\mathrm{xx}}}\right.$, $\overline{\sigma_{y y}}, \overline{\tau_{x y}}$ and $\overline{\tau_{\mathrm{xz}}}$ ) are as follows; $5.23 \%, 6.78 \%, 8.26 \%$, $-21.1 \%$ and $-6.70 \%$. Based on the above percentage differences, it is observed that Murthy results gave closer percentage difference values than that of Ghugal and Sayyad (2010). Results from Reddy (1984) gave percentage differences for in-plane stresses $\left(\overline{\sigma_{y y}}\right.$ and $\left.\overline{\tau_{x y}}\right)$ as $17.73 \%$ and $-22.0 \%$ and also gave percentage differences which agreed better with the present study results for displacement $(\overline{\mathrm{w}})$ and stresses $\left(\overline{\sigma_{\mathrm{xx}}}\right.$ and $\left.\overline{\tau_{\mathrm{xz}}}\right)$ as $4.30 \%, 6.93 \%$ and $-6.23 \%$ respectively. Results from Mindlin (1951) was also compared with the results from present study for displacement $(\overline{\mathrm{W}})$ and stresses $\left(\overline{\sigma_{x x}}, \overline{\sigma_{y y}}, \overline{\tau_{x y}}\right.$ and $\left.\overline{\tau_{x z}}\right)$ and the following percentage differences were obtained; $4.30 \%, 7.23 \%, 18.02 \%$, $20.3 \%$ and $14.74 \%$ respectively. Kirchhoff (1850) results for displacement $(\overline{\mathrm{w}})$ and stresses $\left(\overline{\sigma_{x x}}, \overline{\sigma_{y y}}, \overline{\tau_{x y}}\right.$ and $\overline{\tau_{\mathrm{xz}}}$ ) gave the following percentage differences when compared with the present study; $7.32 \%, 7.23 \%$, $17.73 \%,-20.7 \%$ and $-7.32 \%$. Classical plate theory by Kirchhof over-estimated the in-plane stress $\left(\overline{\sigma_{y y}}\right)$ at percentage difference of $16.84 \%$ but gave the percentage difference for displacement $(\overline{\mathrm{w}})$ and stresses $\left(\overline{\sigma_{\mathrm{xx}}}\right.$ and $\left.\overline{\tau_{\mathrm{xz}}}\right)$ as $4.68 \%, 6.93 \%$ and $-6.23 \%$ respectively. However, from Table-4, average percentage difference for each particular author confirmed that the results obtained by present study are mildly higher or lower when compared with those from previous work and increases or decreases with the same progression. Thus, the solution developed can analyze SSSS and CCCC thick anisotropic plate.

Table-1: Displacements and stresses for SSSS anisotropic rectangular thick plate for $0^{0} @(\alpha=5$ to $100, \beta=$ $\mathbf{b} / \mathbf{a}=2$ )

\begin{tabular}{|r|c|c|c|c|c|c|c|c|}
\hline$\alpha$ & $\overline{\mathbf{w}}$ & $\overline{\mathbf{u}}$ & $\overline{\mathbf{v}}$ & $\overline{\boldsymbol{\sigma}_{\mathbf{x x}}}$ & $\overline{\boldsymbol{\sigma}_{\mathbf{y y}}}$ & $\overline{\boldsymbol{\tau}_{\mathbf{x y}}}$ & $\overline{\boldsymbol{\tau}_{\mathbf{x z}}}$ & $\overline{\boldsymbol{\tau}_{\mathbf{y z}}}$ \\
\hline 5 & 0.13034 & -4.66260 & -2.44529 & 0.66322 & 0.16676 & 0.23516 & 0.63588 & 0.18641 \\
\hline 10 & 0.11934 & -18.5400 & -9.37825 & 0.65758 & 0.16743 & 0.22952 & 0.63921 & 0.19470 \\
\hline 20 & 0.11656 & -74.0417 & -37.1276 & 0.65613 & 0.16765 & 0.22815 & 0.64004 & 0.19689 \\
\hline 30 & 0.11604 & -166.544 & -83.3782 & 0.65586 & 0.16769 & 0.22790 & 0.64020 & 0.19730 \\
\hline 40 & 0.11586 & -296.046 & -148.129 & 0.65577 & 0.16770 & 0.22781 & 0.64025 & 0.19744 \\
\hline 50 & 0.11578 & -462.550 & -231.381 & 0.65572 & 0.16771 & 0.22777 & 0.64028 & 0.19751 \\
\hline 60 & 0.11573 & -666.054 & -333.133 & 0.65570 & 0.16771 & 0.22775 & 0.64029 & 0.19754 \\
\hline 70 & 0.11570 & -906.559 & -453.385 & 0.65568 & 0.16772 & 0.22773 & 0.64030 & 0.19756 \\
\hline 80 & 0.11569 & -1184.06 & -592.138 & 0.65568 & 0.16772 & 0.22772 & 0.64031 & 0.19758 \\
\hline 90 & 0.11567 & -1498.57 & -749.391 & 0.65567 & 0.16772 & 0.22772 & 0.64031 & 0.19759 \\
\hline 100 & 0.11566 & -1850.08 & -925.145 & 0.65566 & 0.16772 & 0.22771 & 0.64031 & 0.19760 \\
\hline
\end{tabular}


Table-2: Displacements and stresses for CCCC anisotropic rectangular thick plate for $0^{0} @(\alpha=5$ to $100, \beta=$ $\mathbf{b} / \mathbf{a}=1.5$ )

\begin{tabular}{|r|c|c|c|c|c|c|c|c|}
\hline $\boldsymbol{\alpha}$ & $\overline{\mathbf{w}}$ & $\overline{\mathbf{u}}$ & $\overline{\mathbf{v}}$ & $\overline{\boldsymbol{\sigma}_{\mathbf{x x}}}$ & $\overline{\boldsymbol{\sigma}_{\mathbf{y y}}}$ & $\overline{\boldsymbol{\tau}_{\mathbf{x y}}}$ & $\overline{\boldsymbol{\tau}_{\mathbf{x z}}}$ & $\overline{\boldsymbol{\tau}_{\mathbf{y z}}}$ \\
\hline 5 & 0.01349 & -0.13438 & -0.26688 & -0.02330 & -0.00145 & 0.03285 & 0.57552 & 0.04710 \\
\hline 10 & 0.00485 & -0.32510 & -0.45571 & -0.01404 & -0.00066 & 0.01549 & 0.63041 & 0.02472 \\
\hline 20 & 0.00244 & -1.06578 & -0.97263 & -0.01147 & -0.00039 & 0.00969 & 0.64738 & 0.01537 \\
\hline 30 & 0.00198 & -2.29763 & -1.79867 & -0.01098 & -0.00034 & 0.00853 & 0.65073 & 0.01338 \\
\hline 40 & 0.00181 & -4.02187 & -2.94989 & -0.01080 & -0.00032 & 0.00811 & 0.65192 & 0.01266 \\
\hline 50 & 0.00174 & -6.23865 & -4.42854 & -0.01072 & -0.00031 & 0.00791 & 0.65247 & 0.01233 \\
\hline 60 & 0.00170 & -8.94801 & -6.23522 & -0.01068 & -0.00030 & 0.00781 & 0.65277 & 0.01214 \\
\hline 70 & 0.00167 & -12.14996 & -8.37012 & -0.01065 & -0.00030 & 0.00774 & 0.65295 & 0.01203 \\
\hline 80 & 0.00166 & -15.84451 & -10.83333 & -0.01064 & -0.00030 & 0.00770 & 0.65307 & 0.01196 \\
\hline 90 & 0.00165 & -20.03166 & -13.62488 & -0.01063 & -0.00030 & 0.00767 & 0.65315 & 0.01191 \\
\hline 100 & 0.00164 & -24.71141 & -16.74480 & -0.01062 & -0.00030 & 0.00765 & 0.65321 & 0.01187 \\
\hline
\end{tabular}

Table-3: Displacements and stresses for CCCC anisotropic rectangular thick plate for $1^{0} @$ $(\alpha=5$ to $100, \beta=b / a=1.5)$

\begin{tabular}{|r|c|c|c|c|c|c|c|c|}
\hline $\boldsymbol{\alpha}$ & $\overline{\mathbf{w}}$ & $\overline{\mathbf{u}}$ & $\overline{\mathbf{v}}$ & $\overline{\boldsymbol{\sigma}_{\mathbf{x x}}}$ & $\overline{\boldsymbol{\sigma}_{\mathbf{y y}}}$ & $\overline{\boldsymbol{\tau}_{\mathbf{x y}}}$ & $\overline{\boldsymbol{\tau}_{\mathbf{x z}}}$ & $\overline{\boldsymbol{\tau}_{\mathbf{y z}}}$ \\
\hline 5 & 0.01704 & -0.16094 & -0.31905 & -0.31784 & -0.02747 & 0.16179 & 0.74015 & 0.07032 \\
\hline 10 & 0.00518 & -0.34977 & -0.45589 & -0.13168 & -0.01129 & 0.06619 & 0.66805 & 0.04452 \\
\hline 20 & 0.00258 & -1.14655 & -0.99602 & -0.08639 & -0.00734 & 0.04286 & 0.65355 & 0.03529 \\
\hline 30 & 0.00211 & -2.47837 & -1.88603 & -0.07787 & -0.00660 & 0.03846 & 0.65105 & 0.03334 \\
\hline 40 & 0.00194 & -4.34341 & -3.13019 & -0.07487 & -0.00634 & 0.03691 & 0.65019 & 0.03263 \\
\hline 50 & 0.00187 & -6.74147 & -4.72927 & -0.07348 & -0.00621 & 0.03619 & 0.64979 & 0.03230 \\
\hline 60 & 0.00183 & -9.67248 & -6.68348 & -0.07273 & -0.00615 & 0.03580 & 0.64958 & 0.03212 \\
\hline 70 & 0.00180 & -13.13643 & -8.99290 & -0.07227 & -0.00611 & 0.03557 & 0.64945 & 0.03202 \\
\hline 80 & 0.00179 & -17.13330 & -11.65757 & -0.07198 & -0.00608 & 0.03542 & 0.64936 & 0.03194 \\
\hline 90 & 0.00178 & -21.66310 & -14.67749 & -0.07177 & -0.00607 & 0.03531 & 0.64931 & 0.03190 \\
\hline 100 & 0.00177 & -26.72582 & -18.05267 & -0.07163 & -0.00605 & 0.03524 & 0.64926 & 0.03186 \\
\hline
\end{tabular}

Table-4: Comparison of deflection and stresses from present study with those of Ghugal and Sayyad (2010), Murty(1984), Reddy(1984), Mindlin(1951) Kirchhoff(1850) and Classical Plate for rectangular isotropic plate at $0^{0}$ angle fiber orientation

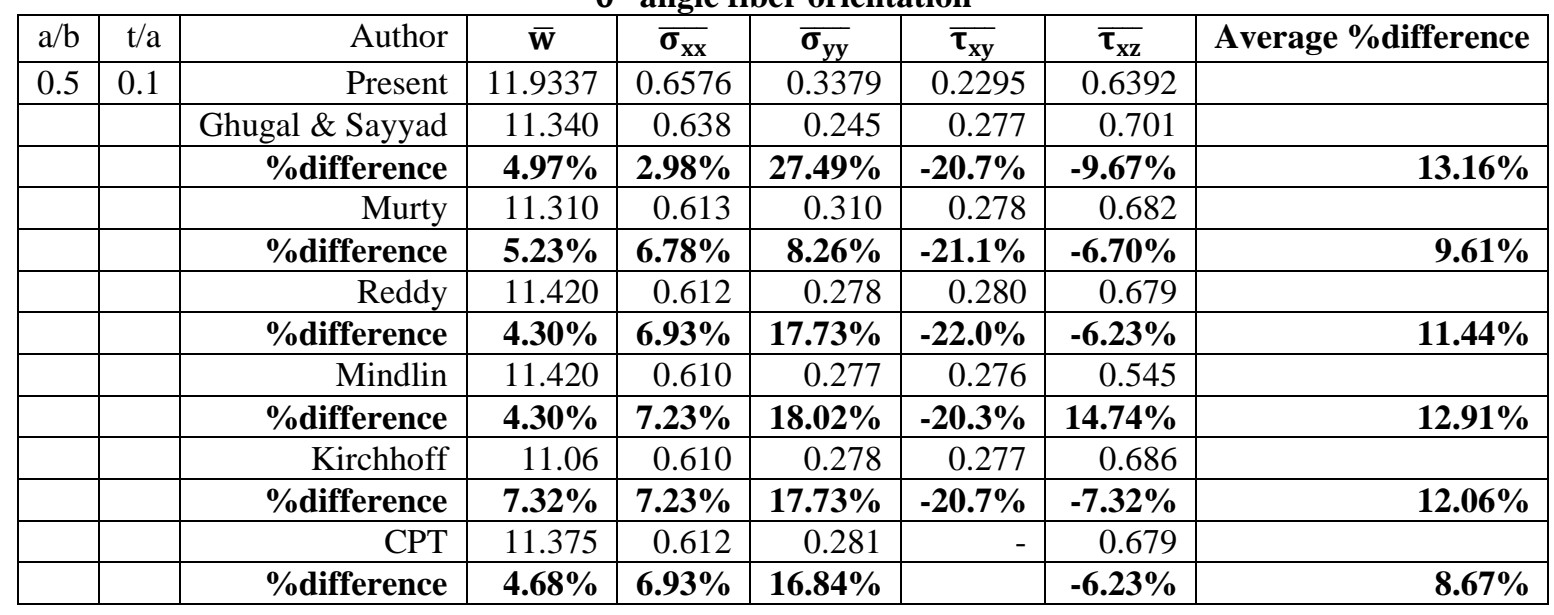

\section{CONCLUSIONS}

The study presents a solution for the analysis of SSSS and CCCC thick rectangular anisotropic plates. From the governing equation, compatibility equations and based on the third order shear deformation theory, the solution applied Ritz energy method to derived the polynomial displacement functions for SSSS and CCCC rectangular plate. Deflection at the center of the anisotropic rectangular plate were determined for $0^{0}$, $15^{0}$ and for span to thickness ratio, alpha, $(\alpha=5,10,20$,
30, 40, 50, 60, 70, 80, 90 and 100) for SSSS and CCCC thick anisotropic rectangular plate. The displacements ( $\mathrm{u}$ and $\mathrm{v}$ ) and stresses $\left(\sigma_{\mathrm{x}}, \sigma_{\mathrm{y}}, \tau_{\mathrm{xy}}, \tau_{\mathrm{xz}}\right.$ and $\left.\tau_{\mathrm{yz}}\right)$ were also determined in accordance to the description above. From the numerical results obtained, the following conclusions were drawn.

The solutions developed through exact approach can be used for satisfactory analysis to anisotropic thick rectangular plate problems of SSSS 
and CCCC boundary conditions. Also, the exact approach of analyzing anisotropic thick rectangular plate using third order shear deformation theory in Ritz energy method, yielded satisfactory numerical results when compared with those from previous studies.

\section{REFERENCES}

1. Sarangan, S., \& Singh, B. N. (2017). Evaluation of free vibration and bending analysis of laminated composite and sandwich plates using non-polynomial zigzag models: COFinite element formulation. Journal of Aerospace Science and Technology, 68(6):496-508. https://doi.org/10.1016/j.ast.2017.06.001

2. Zhanga, H., Shia, D., \& Wangb, Q. (2017). An improved Fourier series solution for free vibration analysis of the moderately thick laminated composite rectangular plate with non-uniform boundary onditions. International Journal of Mechanical Science. 121(11):1-20. https://doi.org/10.1016/j.ijmecsci.2016.12.007

3. Iyengar, K. T. S., \& Pandya, S. K. (1983). Analysis of orthotropic rectangular thick plates. Fibre Science and Technology. 18(1):19-36. https://doi.org/10.1016/0015-0568(83)90048-9

4. Sciuva, M. (1986). Bending, vibration and buckling of simply supported thick multilayered orthotropic plates: An evaluation of a new displacement model. 105(3):425-442. https://doi.org/10.1016/0022460X(86)90169-0

5. Aydogdu, M. (2009). A new shear deformation theory for laminated composite plates. Composite Structures. 89(7):94-101. http://dx.doi.org/10.1016/j.compstruct.2008.07.008

6. Noor, A. K., \& Burton, W. S. (1989). Assessment of shear deformation theories for multilayered composites Plates. Applied Mechanics Rev. 42(1):113. http://dx.doi.org/10.1115/1.3152418

7. Reddy, J., \& Robbins, D. (1994). Theories and computational models for composite laminates. Applied Mechanics Rev. 47(1):147-169. http://dx.doi.org/10.1115/1.3111076

8. Robbins, D. H., \& Reddy, J. N. (1993). Modelling of thick composites using a layerwise laminate theory. International Journal of Numerical Methods in Engineering. 36(1):655-677. http://dx.doi.org/10.1002/nme.1620360407

9. Zhang, Y. X., \& Yang, C. H. (2009). Recent developments in finite element analysis for laminated composite plates. Composite Structures. 88(2):147157. http://dx.doi.org/10.1016/j.compstruct.2008.02.014

10. Khandan, R., Noroozi, S., Sewell, P., \& Vinney, J. (2012). The development of laminated composite plate theories: A review. Journal of Material Science. $47(12), \quad 5901 \quad-\quad 5910$. http://dx.doi.org/10.1007/s10853-012-6329-y

11. Carrera, E. (2002). Theories and finite elements for multilayered, anisotropic, composite plates and shells. Archive of Computer Methods in Engineering 9(1):87-140. http://dx.doi.org/10.1007/BF02736649

12. Matsunaga, H. (2002). Assessment of global higherorder deformation theory for laminated composite and sandwich plates. Composites Structures. 56(2):279-291. http://dx.doi.org/10.1016/S02638223(02)00013-2

13. Reddy, J. (1984). A simple higher-order theory for laminated composite plates. Journal of Applied Mechanics. http://dx.doi.org/10.1115/1.3167719

14. Cho, M., \& Parmerter, R. (1993). Efficient higher order composite plate theory for general lamination configurations. American Institute of Aeronautics and Astronautics. 31(7):1299-1306. https://doi.org/10.2514/3.11767

15. Poniatovski, V. V. (1964). On the theory of bending of anisotropic plates. Journal of applied Mathematics and Mechanics. 28(6):1247-1254. https://doi.org/10.1016/0021-8928(64)90036-X

16. Illing, E. (1952). The bending of thin anisotropic plates. The Quarterly Journal of Mechanics and Applied Mathematics. 5(1):12-28. https://doi.org/10.1093/qjmam/5.1.12

17. Vijayakumar, K. (2013). Poisson's theory for analysis of bending of isotropic and anisotropic plates. International scholarly research notices. 1-8. https://doi.org/10.1155/2013/562482

18. Vasilenko, A. T. (2002). Bending of an anisotropic elliptic plate on an elastic foundation. International Applied Mechanics. 38:351-355. https://doi.org/10.1023/A:1016042530247

19. Hearmon, R. F. S., \& Adams, E. H. (1952). The bending and twisting of anisotropic plates. British Journal of Applied Physics, 3(5):1-22. https://doi.org/10.1088/0508-3443/3/5/305

20. Gholami, M., Hassani, A., Ojarestaghi, S. S. M., \& Alashti, R. A. (2019). Bending analysis of anisotropic functionally graded plates based on three dimensional elasticity. Article is under review in European Journal of Computational Mechanics. https://doi.org/10.1080/17797179.2018.1560844

21. Ghugal, Y. M., \& Sayyad, A. S. (2010). A static flexure of thick isotropic plates using trigonometric shear deformation theory. Journal o Solid Mechanics, 2(1), 79-90. https://doi.org/wwwjsm.paper.pdf

22. Murty, A. V. K. (1984). Toward a consistent beam. American Institute of Aeronautics and Astronautics AIAA Journal, 22(1):811-816. https://www.eprints.library.lisc.ernet.in

23. Reddy J. N. (2004). Mechanics of laminated composite plates and shell "theory and analysis" (Second edition). CRC Press: Washington D.C.

24. Mindlin, R. D. (1951). Influence of rotary inertia and shear on flexural motions of isotropic elastic plates. Journal of Applied Mechanics, 18, 31-38.

25. Kirchhoff, G. R. (1850). Uber das gleichgewichi und die bewegungeinerelastishemscheibe. Journal Fuer die Reine und Angewandte Mathematik, 40, 5188 https://doi.org/10.1515/crit.1850.40.51. 